\title{
The effect of graft choice on functional outcome in anterior cruciate ligament reconstruction
}

\author{
Matjaž Sajovic • Andrej Strahovnik • \\ Radko Komadina $\cdot$ Mojca Z. Dernovšek
}

Received: 9 January 2007 / Accepted: 16 January 2007 / Published online: 13 March 2007

(C) Springer-Verlag 2007

\begin{abstract}
A prospective, randomised, 5-year follow-up study was designed to compare the functional results between patellar tendon and hamstring tendon autografts after anterior cruciate ligament reconstruction. Primary reconstruction was performed in 32 patients using the central third of the patellar ligament and in 32 patients using double-looped semitendinosus and gracilis tendons. All reconstructions were performed by a single surgeon, with identical surgical technique and rehabilitation protocol. Of the total 64 patients in the study, $54(85 \%)$ were available for the 5-year follow-up. No statistically significant differences were seen with respect to Lysholm score, International Knee Documentation Committee (IKDC) classification, clinical and KT-2000 arthrometer laxity testing, single-legged hop test and anterior knee pain. Graft rupture occurred in two patients $(8 \%)$ in the patellar tendon group and in two patients $(7 \%)$ in the hamstring tendon group; 23 patients $(88 \%)$ in the patellar tendon group and 23 patients (82\%) in the hamstring tendon group returned to their pre-injury activity level. Good subjective outcome and stability can be obtained by using either graft; no
\end{abstract}

M. Sajovic · A. Strahovnik $(\bowtie)$

Department of Orthopaedics and Sports Injuries,

General Hospital Celje,

Oblakova 5,

3000 Celje, Slovenia

e-mail: andrej_strahovnik@yahoo.com

R. Komadina

Department of Traumatology, General Hospital Celje, Celje, Slovenia

M. Z. Dernovšek

Institute of Public Health of the Republic of Slovenia, Ljubljana, Slovenia statistically significant differences were found in functional outcome between the grafts.

Résumé Une étude prospective randomisée avec un suivi moyen de 5 ans a été réalisée de façon à comparer les résultats de la reconstruction du ligament croisé antérieur par tendon rotulien ou tendon de la patte d'oie. Cette reconstruction a bénéficié à 32 patients, dans chaque type de traitement. Pour les patients traités par tendons de la patte d'oie on a utilisé à l'aide d'un double renfort le semitendineux et le gracilis. Toutes ces interventions ont été réalisées par le même chirurgien avec la même technique de rééducation. Sur les 64 patients de l'étude, 54 (85\%) ont pu être suivis pendant 5 ans. Il n'y a pas de différence significative entre ces deux séries, après analyse selon le score de Lysholm, la classification IKDC, la laxité clinique et la laxité évaluée par le KT-2000. De même en ce qui concerne le sautillement et les douleurs antérieures du genou. Une rupture de la greffe est survenue chez deux patients $(8 \%)$ du groupe tendon rotulien et chez deux patients (7\%) du groupe tendon de la patte d'oie. 23 patients $(88 \%)$ du groupe tendon rotulien et 23 patients $(82 \%)$ du groupe patte d'oie ont pu reprendre leurs activités à un niveau identique à celui qu'ils avaient avant l'accident. Ces techniques permettent d'obtenir une bonne stabilité sur le plan résultats subjectifs sans différence significative quelle que soit la greffe utilisée.

\section{Introduction}

The rupture of the anterior cruciate ligament (ACL) is the most common severe injury of the knee [7]. The higher participation of the population in sports has resulted in a constantly increasing incidence of ACL tears. The func- 
tional instability after the rupture can lead to additional meniscal and chondral injuries as well as to the development of degenerative joint disease $[9,15,16,22]$. Successful treatment must therefore preserve intact meniscal and chondral structures. The goals of ACL reconstruction are to provide a functionally stable knee, decrease symptoms and return patients to their pre-injury activity level [21].

Patellar tendon and hamstring tendon autografts are the most commonly used for ACL reconstruction. The advantage of the patellar tendon autograft is faster and stronger bone-to-bone ingrowth, whereas the disadvantages are the possibility of patellar fracture, a potential increase in patellofemoral pain and rupture of the patellar tendon [3, 13, 14]. Using a hamstring tendon autograft can result in slower healing of the graft attachment site and hamstring muscle weakness [4, 25]. The biomechanical properties of patellar tendon and double-looped hamstring tendons autografts are comparable on strength examinations [19].

There are few prospective randomised studies on ACL reconstruction directly comparing the outcome of both grafts $[1,2,5,6,11,18,20]$. The purpose of this prospective, randomised, 5-year follow-up study is to compare the functional results after arthroscopic single-incision ACL reconstruction with patellar tendon or four-strand hamstring tendon autografts, both fixed with interference screws by the same surgeon using the same surgical technique and post-operative rehabilitation.

\section{Materials and methods}

Patients with clinically diagnosed ACL rupture and inability to return to pre-injury activity level were operated upon between June 1999 and March 2000 in our department. Exclusion criteria included associated ligament injury, previous meniscectomy, abnormal contralateral knee and patients' unwillingness to participate in a research programme. Also, patients who required either revision surgery or primary surgery of the contralateral knee during the follow-up were excluded from the study. All patients who fulfilled the inclusion criteria signed an informed consent form. The study was approved by the National Ethics Committee.

A group of 64 patients was initially formed. Patients were randomised into two groups of equal sizes according to operative registration list position, distributing patients with even numbers into the patellar tendon group (PT group) and patients with odd numbers into the hamstring tendon group (STG group). A primary arthroscopically assisted ACL reconstruction was performed in both groups with 32 patients each by the first author using the same surgical technique except for the autograft.
All patients were operated under general anaesthesia and meniscal surgery was performed, if needed, prior to the ACL reconstruction. The menisci were sutured with nonabsorbable sutures using the inside-out technique. Drill guides were used to confirm the correct position of the tunnels. The femoral tunnel was drilled through the anteromedial portal before tibial tunnel drilling. The graft was inserted retrograde via the tibial tunnel into a blind femoral tunnel. The femoral graft fixation was achieved with round head, cannulated interference screws (RCI, ART-MAM, Slovenia), designed by the first author. In right knees the screw with left thread and in left knees the screw with right thread were used so as to prevent graft impingement against the lateral wall of the intercondylar notch. The tibial graft fixation was attained with bioabsorbable interference screws (Linvatec Biomaterials, Tampere, Finland) in an outside-in direction at a knee flexion angle of approximately $10^{\circ}$ and manual pretension.

In the PT group, the surgical procedure was an arthroscopic single-incision technique with the central third of the ipsilateral bone-patellar-bone tendon used as a free autograft. The defects of the patella and the proximal tibia were not bone grafted. Tunnel size in the PT group was determined to be $1 \mathrm{~mm}$ larger than the bone block size.

In the STG group, the surgical procedure was an arthroscopic single-incision technique with double-looped semitendinosus and gracilis tendons. The drill tunnels were 8 or $9 \mathrm{~mm}$ in diameter and were equal to the cross-sectional size of the graft. A marking suture using No. 0 absorbable suture was set $2.5 \mathrm{~cm}$ from the femoral end of the graft to ensure good entry of the graft into the tunnel and to prevent the graft from twisting around the screw during insertion.

All patients received rehabilitation therapy according to the same accelerated protocol, which permitted immediate full weight bearing and full range of motion [22]. A rehabilitation brace was used for 3 weeks post-operatively. No splints were worn during the rehabilitation protocol or during the night. The importance of reaching full extension was emphasised from the beginning. The patients had to obtain an arc of at least $0-90^{\circ}$ before they were discharged from the hospital. Closed kinetic chain exercises were started immediately after the operation. Resisted extension exercises between $45-0^{\circ}$ were not allowed during the first 6 post-operative weeks. Running was allowed at 8 weeks and contact sports at 6 months under three conditions: no effusion, full range of motion and obtained muscle strength of $90 \%$ compared with the contralateral side.

The Lysholm knee score questionnaire [24] was sent to the patients by post and they responded in their home environment. The International Knee Documentation Committee (IKDC) activity level was used to assess the functional outcome [10, 25]. Range of motion was measured to the nearest $5^{\circ}$ by using a goniometer and bony landmarks 
at the lateral malleolus of the ankle, knee joint line and greater trochanter of the hip. Clinical ligament testing was performed by means of the Lachman test, anterior drawer test and pivot shift test, with side-to-side differences recorded. Objective anteroposterior knee stability was determined by using the KT-2000 arthrometer (MEDmetric, San Diego, CA, USA) at 89 and $134 \mathrm{~N}$ manual tension and a knee flexion angle of $25^{\circ}$. We selected $3 \mathrm{~mm}$ as the upper limit of normality of side-to-side difference in anterior tibial displacement. The manual maximum displacement test was not relevant, because the physiotherapist had not been able to produce a greater force than $134 \mathrm{~N}$ and fix the patella at the same time. After the evaluation of the IKDC score, we graded these parameters as A (normal), B (nearly normal), C (abnormal) and D (severely abnormal) compared with the patients' pre-operative conditions or the control knees.

The patients were classified as having subjective anterior knee pain if they registered pain during or after activity, during stair walking and during squatting or kneeling. The single-legged hop test was used to evaluate functional performance, with grade A hop given to patients whose hopping distance was equal or greater than $90 \%$ of a distance achieved on the contralateral limb.

Median (range) values are presented, except for the absolute anterior KT-2000 arthrometer laxity measurements, for which mean (range) values are presented. The paired $t$-test was used for comparisons of the pre-operative and post-operative numerical data (KT-2000 arthrometer, range of motion, Lysholm score), and unpaired $t$-test for numerical data within the groups, respectively. The chisquare test was used to compare categorical variables (overall IKDC scores). A $p$ value less than 0.05 was considered statistically significant.

\section{Results}

Of the 32 patients in each group at the beginning of the study, 6 patients in the PT group and 4 patients in the STG group were excluded during the follow-up period, leaving 54 patients $(85 \%)$ available for analysis. Two patients in the PT group and one in the STG group were lost to follow-up; the remaining seven patients were excluded due to a contralateral ACL rupture or revision ACL surgery of the knee. Three PT and two STG patients suffered a contralateral ACL rupture during the study period. One male patient in the PT group had a beach volleyball reinjury while one female patient in the STG group had a skiing reinjury of the reconstructed knee; thus, revision ACL reconstruction had been performed in both patients. In the end, 26 patients in the PT group and 28 patients in the STG group were clinically examined at 5 years follow-up.

There were no significant differences in demographics (age, gender), pre-injury activity level, pre-operative Lysholm knee score or interval from injury to surgery in both groups (Table 1).

An injury of medial meniscus at the time of reconstruction was found in 12 of 26 PT patients $(46 \%)$ and in 12 of 28 STG patients (43\%). A lateral meniscal injury was observed in 6 of PT patients (23\%) and in 6 of STG patients $(21 \%)$. Meniscal suturing was performed in one patient in the PT group (4\%) and in two patients in the STG group (7\%). Subtotal meniscal resections were performed in 4 of 26 PT patients (15\%) and in 12 of 28 STG patients (43\%); the difference between the two groups was, unfortunately, statistically significant ( $p=0.027$ ).

The median length of hospital stay was 4 nights (range: $2-$ 6) for the PT group and three nights (range: $2-4$ ) for the STG group. There were no infections, deep venous thromboses,

Table 1 Comparison of randomised groups

\begin{tabular}{lll}
\hline & Hamstring tendon group & Patellar tendon group \\
\hline Total number of patients & 28 & 26 \\
Age, years (range) & $24(14-42)$ & $27(16-46)$ \\
Gender & & 14 \\
Male & 13 & 12 \\
Female & 15 & 18 \\
IKDC pre-injury activity level & & $55(16-77)$ \\
Level I & 18 & $23(1-60)$ \\
Pre-operative Lysholm scores, mean (range) & $57(9-82)$ & $4(15 \%)$ \\
Time from injury to surgery, months, mean (range) & $25(1-84)$ & $22(85 \%)$ \\
Acute reconstruction $(n)$ & $4(14 \%)$ & $19(73 \%)$ \\
Chronic reconstruction $(n)$ & $24(86 \%)$ & $1(4 \%)$ \\
Meniscal surgery $(n)$ & $20(71 \%)$ & $14(54 \%)$ \\
Meniscal repair $(n)$ & $2(7 \%)$ & $4(15 \%)$ \\
Partial resection $(n)$ & $6(21 \% 9)$ & 0.611 \\
Subtotal resection $(n)$ & $12(43 \%)$ & \\
\hline
\end{tabular}


nerve injuries, arthrofibrosis, impingement syndrome or other complications in the series.

In the PT group, there were two cases of graft ruptures and three patients with ruptured contralateral ACL. In the STG group, two patients had graft rupture, and two patients ruptured their contralateral ACL. The differences in the rate of ACL graft rupture and contralateral ACL rupture were not statistically significant.

A significant improvement comparing pre-operative and post-operative Lysholm knee scores was seen in both groups (PT: $p<0.001$; STG: $p<0.001$ ). However, no significant difference in Lysholm score between the groups was observed at 5 years follow-up (Table 2). The mean Lysholm score in the PT group was 92 (range: 62-100) and in the STG group 92 (range: 74-100).

Table 2 also represents the distribution of patients according to overall IKDC score. Grade A was obtained in $38 \%$ of patients in the PT group and in $50 \%$ of patients in the STG group. The difference between the groups was also not statistically significant $(p=0.692)$.

There were 23 patients (88\%) in the PT group and 23 patients $(82 \%)$ in the STG group who returned to their preinjury activity level. On a single-legged hop test, a grade A hop was achieved by 24 patients $(92 \%)$ in the PT group and 26 patients $(93 \%)$ in the STG group.

The anterior knee pain and kneeling pain was a minor problem. There were $19 \%$ of patients in the PT group and $17 \%$ in the STG group who reported anterior knee pain during strenuous activities or in a kneeling-squatting position. The range of motion of the reconstructed knee was physiological compared to the healthy contralateral limb in both groups. Extension was full in all patients whereas three patients (11\%) in the PT group had a $10^{\circ}$ lack of terminal passive flexion.

Table 2 Lysholm knee scores and overall IKDC score 5 years postoperatively

\begin{tabular}{|c|c|c|c|c|c|}
\hline & \multirow{2}{*}{\multicolumn{2}{|c|}{$\begin{array}{l}\begin{array}{l}\text { Hamstring } \\
\text { tendon group }\end{array} \\
(n=28)\end{array}$}} & \multirow{2}{*}{\multicolumn{2}{|c|}{$\begin{array}{l}\begin{array}{l}\text { Patellar } \\
\text { tendon group }\end{array} \\
(n=26)\end{array}$}} & \\
\hline & & & & & \\
\hline & $n$ & $\%$ & $n$ & $\%$ & \\
\hline Lysholm & & & & & $p=0.888$ \\
\hline Excellent (95-100) & 16 & 58 & 11 & 43 & \\
\hline Good (84-94) & 9 & 32 & 13 & 50 & \\
\hline Fair (65-83) & 3 & 10 & 2 & 7 & \\
\hline Poor $(<65)$ & 0 & 0 & 0 & 0 & \\
\hline IKDC & & & & & $p=0.692$ \\
\hline $\mathrm{A}$ & 14 & 50 & 10 & 38 & \\
\hline $\mathrm{B}$ & 13 & 47 & 15 & 59 & \\
\hline $\mathrm{C}$ & 1 & 3 & 1 & 3 & \\
\hline $\mathrm{D}$ & 0 & 0 & 0 & 0 & \\
\hline
\end{tabular}

Table 3 Outcomes of clinical evaluations

\begin{tabular}{|c|c|c|c|c|c|}
\hline & \multicolumn{2}{|c|}{$\begin{array}{l}\text { Hamstring } \\
\text { tendon } \\
\text { group }\end{array}$} & \multicolumn{2}{|c|}{$\begin{array}{l}\text { Patellar } \\
\text { tendon } \\
\text { group }\end{array}$} & \multirow[b]{2}{*}{$p$} \\
\hline & $n$ & $\%$ & $n$ & $\%$ & \\
\hline \multicolumn{6}{|l|}{ Manual Lachman test } \\
\hline $\mathrm{A}$ & 22 & 79 & 22 & 85 & \\
\hline $\mathrm{B}$ & 5 & 18 & 3 & 12 & \\
\hline $\mathrm{C}$ & 1 & 3 & 1 & 3 & \\
\hline \multicolumn{6}{|l|}{ Manual pivot shift test } \\
\hline A & 23 & 83 & 21 & 81 & \\
\hline $\mathrm{B}$ & 4 & 14 & 4 & 19 & \\
\hline $\mathrm{C}$ & 1 & 3 & 1 & 3 & \\
\hline $\begin{array}{l}\text { IKDC instrumental anteroposterior } \\
\text { translation (KT-2000 arthrometer, } \\
25^{\circ} \text { flexion) }\end{array}$ & & & & & 0.646 \\
\hline $\mathrm{A}(0-2 \mathrm{~mm})$ & 22 & 80 & 21 & 82 & \\
\hline $\mathrm{B}(3-5 \mathrm{~mm})$ & 5 & 17 & 4 & 15 & \\
\hline $\mathrm{C}(6-10 \mathrm{~mm})$ & 1 & 3 & 1 & 3 & \\
\hline $\mathrm{D}(>10 \mathrm{~mm})$ & 0 & 0 & 0 & 0 & \\
\hline
\end{tabular}

One patient in each group had a positive pivot shift test (Table 3). We could not find any objective explanation for the graft failures. The time from injury to operation was 5 months in patients in the PT group and 6 months in patients in the STG group.

Using the KT-2000 arthrometer and IKDC reference, patients were classified according to their post-operative anterior laxity into four groups (Table 3). The mean value of anterior laxity measured at $134 \mathrm{~N}$ of manual tension (side-to-side difference) was $1.9 \pm 2.0 \mathrm{~mm}$ for the PT group and $1,6 \pm 2,4 \mathrm{~mm}$ for the STG group ( $p=0.646)$. In the PT group, $76 \%$ (20 of 26 ) scored $3 \mathrm{~mm}$ or less difference, as did $78 \%$ (22 of 28 ) of the STG group. One patient (3\%) in the PT group and one (3\%) in the STG group scored more than $5 \mathrm{~mm}$. Abnormally high $\mathrm{KT}$ results $(7 \mathrm{~mm}$ and $9 \mathrm{~mm}$ ) in two patients were reflected by the clinical examination and patients' subjective opinion.

\section{Discussion}

In a randomised, prospective study, we compared the longterm functional results in two groups of patients, whose treatment differed in the choice of the autograft. Apart from the graft, all other important factors for the clinical outcome were identical. All reconstructions were performed by one surgeon; the fixation technique and the rehabilitation protocol were the same. That is the strength of our study. Numerous studies have been published comparing patellar and hamstring tendon autografts in arthroscopic ACL reconstruction $[1,2,5$, $6,11,18,20]$. Many of them have been prospective and 
randomised, but direct comparison between the grafts was not reliable because fixation techniques, surgical techniques and rehabilitation protocols differed [7, 11].

The drawback of our study, as of many other studies, is the inclusion of patients with additional meniscal injuries. It is difficult to obtain enough patients with solitary anterior cruciate ligament tear without any other intra-articular lesion.

The results of our study confirm the findings from previous reports that good or excellent results may be obtained in the majority of ACL reconstructions using either patellar or hamstring tendon autografts. The overall results of this study, according to the IKDC evaluation system, were $97 \%$ of patients with normal or nearly normal knee in the patellar and hamstring tendon groups. This result is high in comparison to the results of other prospective studies $[2,5,6,11,18,20]$. The overall IKDC results in our study may be so high due to the limited number of patients. The comparison between the groups with respect to Lysholm and IKDC score did not show any statistically significant differences. There was only a trend to better functional outcome in the hamstring tendon group, as patients from this group were more likely to achieve the grade "excellent" and grade "A".

Roe et al. [18] compared the grafts in a prospective clinical study with the longest follow-up of 7 years. In contrast to our study, their study eliminated all patients with more than one-third meniscectomy. Both studies excluded the patients with contralateral ACL rupture and patients with revision surgery during the follow-up. Their study, however, did not include the patients with atraumatic graft failures. Nonetheless, neither of the studies represents a significant difference in overall rate of ACL rupture.

Loss of extension after ACL reconstruction had been linked to the harvesting of the central third of the patellar tendon. Pinczewski et al. [17] reported that $31 \%$ of patients in the patellar tendon group had an extension deficit whereas only $19 \%$ of the hamstring tendon group had extension deficit at 5 years follow-up. Goldblatt et al. [8] in his meta-analysis found a trend toward a loss of extension in patellar tendon groups while the patients in hamstring tendon groups were more likely to lose flexion. Our results demonstrate no statistically significant difference in range of motion between the groups.

Anterior knee pain has also been frequently reported as a major concern with the patellar tendon autograft. Several authors have shown that the use of hamstring tendon causes less post-operative donor site morbidity and anterior knee pain [5]. Marder et al. [12] reported a $24 \%$ incidence of anterior knee pain in patients with either autograft. Similar to our study, however, Aglietti et al. [1] and Anderson et al. [2] found anterior knee pain to be only a minor problem. We also did not find a difference in the number of donor site symptoms between the groups. Shelbourne and
Trumper [23] compared a group of 602 patients after reconstruction with the patellar tendon autograft to a control group of 122 patients who had no previous knee injury. They reported little or no anterior knee pain in both groups and concluded that it is probably not an inherent complication associated with patellar tendon harvesting. They suggested that the incidence of anterior knee pain can be lowered by obtaining early full knee hyperextension after the operation.

We used Lachman, drawer, pivot shift test and KT-2000 side-to-side measurements to assess stability and found no significant differences. Many investigations showed better stability when patellar tendon graft was used $[1,2,5,17]$.

In conclusion, the results of our study substantiate the similarities between the grafts found in previous reports. Good or excellent results can be obtained in the majority of ACL reconstructions using either patellar tendon or hamstring tendon autografts. No significant differences in the rate of graft failure or contralateral ACL rupture were identified. Both grafts provided good subjective outcomes and objective stability at 5 years.

\section{References}

1. Aglietti P, Buzzi R, Zaccherotti G, De Biase P (1994) Patellar tendon versus doubled semitendinosus and gracilis tendons for anterior cruciate ligament reconstruction. Am J Sports Med 22:211-218

2. Anderson AF, Snyder RB, Lipscomb AB Jr (2001) Anterior cruciate ligament reconstruction: a prospective randomized study of three surgical methods. Am J Sports Med 29:272-279

3. Bonamo JJ, Krinick RM, Sporn AA (1984) Rupture of the patellar ligament after use of its central third for anterior cruciate reconstruction. A report of two cases. J Bone Joint Surg Am 66:1294-1297

4. Coombs R, Cochrane T (2001) Knee flexor strength following anterior cruciate ligament reconstruction with the semitendinosus and gracilis tendons. Int J Sports Med 22:618-622

5. Ejerhed L, Kartus J, Sernert N, Kohler K, Karlsson J (2003) Patellar tendon or semitendinosus tendon autografts for anterior cruciate ligament reconstruction? A prospective randomized study with a two-year follow-up. Am J Sports Med 31:19-25

6. Feller JA, Webster KE (2003) A randomized comparison of patellar tendon and hamstring tendon anterior cruciate ligament reconstruction. Am J Sports Med 31:564-573

7. Freedman KB, D'Amato MJ, Nedeff DD, Kaz A, Bach BR Jr (2003) Arthroscopic anterior cruciate ligament reconstruction: a metaanalysis comparing patellar tendon and hamstring tendon autografts. Am J Sports Med 31:2-11

8. Goldblatt JP, Fitzsimmons SE, Balk E, Richmond JC (2005) Reconstruction of the anterior cruciate ligament: meta-analysis of patellar tendon versus hamstring tendon autograft. Arthroscopy 7:791-803

9. Hawkins RJ, Misamore GW, Merritt TR (1986) Follow-up of the acute nonoperated isolated anterior cruciate ligament tear. Am J Sports Med 14:205-210

10. Hefti F, Muller W, Jakob RP, Staubli HU (1993) Evaluation of knee ligament injuries with the IKDC form. Knee Surg Sports Traumatol Arthrosc 1:226-234 
11. Jansson KA, Linko E, Sandelin J, Harilainen A (2003) A prospective randomized study of patellar versus hamstring tendon autografts for anterior cruciate ligament reconstruction. Am J Sports Med 31:12-18

12. Marder RA, Raskind JR, Carrol M (1991) Patellar tendon versus double semitendinosus and gracilis tendons for anterior cruciate ligament reconstruction. Am J Sports Med 19:478-484

13. Marumoto JM, Mitsunaga MM, Richardson AB, Medoff RJ, Mayfield GW (1996) Late patellar tendon ruptures after removal of the central third for anterior cruciate ligament reconstruction: a report of two cases. Am J Sports Med 24:698-701

14. Mastrokalos DS, Springer J, Siebold R, Paessler HH (2005) Donor site morbidity and return to the preinjury activity level after anterior cruciate ligament reconstruction using ipsilateral and contralateral patellar tendon autograft. Am J Sports Med 33:85-93

15. McDaniel WJ Jr, Dameron TB Jr (1980) Untreated ruptures of the anterior cruciate ligament. A follow-up study. J Bone Joint Surg Am 62:696-705

16. Noyes FR, Mooar PA, Mathews DS, Butler DL (1983) The symptomatic anterior cruciate-deficient knee. Part I: the long-term functional disability in athletically active individuals. J Bone Joint Surg Am 65:154-162

17. Pinczewski LA, Deehan DJ, Salmon LJ, Russel VJ, Clingeleffer A (2002) A five-year comparison of patellar tendon versus fourstrand hamstring tendon autograft for arthroscopic reconstruction of the anterior cruciate ligament. Am J Sports Med 30:523-536
18. Roe J, Pinczewski LA, Russell VJ, Salmon LJ, Kawamata T, Chew M (2005) A 7-year follow-up of patellar tendon and hamstring tendon grafts for arthroscopic anterior cruciate ligament reconstruction. Am J Sports Med 33:1337-1345

19. Rowden NJ, Sher D, Rogers GJ, Schindhelm K (1997) Anterior cruciate ligament graft fixation: initial comparison of patellar tendon and semitendinosus autografts in young fresh cadavers. Am J Sports Med 25:472-478

20. Shaieb MD, Kan DM, Chang SK, Marumoto JM, Richardson AB (2002) A prospective randomized comparison of patellar tendon versus semitendinosus and gracilis tendon autografts for anterior cruciate ligament reconstruction. Am J Sports Med 30:214-220

21. Shelbourne KD, Gray T (2000) Results of anterior cruciate ligament reconstruction based on meniscus and articular cartilage status at the time of surgery. Five- to fifteen-year evaluations. Am J Sports Med 28:446-452

22. Shelbourne KD, Nitz P (1990) Accelerated rehabilitation after anterior cruciate ligament reconstruction. Am J Sports Med 18:292-299

23. Shelbourne KD, Trumper RV (1997) Preventing anterior knee pain after anterior cruciate ligament reconstruction. Am J Sports Med 25:41-47

24. Tegner Y, Lysholm J (1985) Rating systems in the evaluation of the knee ligament injuries. Clin Orthop 198:43-49

25. West RV, Harner CD (2005) Graft selection in anterior cruciate ligament reconstruction. J Am Acad Orthop Surg 13:197-207 